\title{
Relación entre la depreciación implícita en el mercado de bonos gubernamentales y el mercado bancario en República Dominicana
}

\author{
Por EnRique Penson*
}

Recibido: 29/1/2017 - Aceptado: 1/8/2017

\begin{abstract}
Resumen
En este artículo estimo la relación de causalidad entre la depreciación implícita del tipo de cambio peso/dólar en las transacciones de bonos gubernamentales en el mercado de bonos y la depreciación efectiva reflejada en las operaciones de la banca comercial. Para hacer esto, se extrajo la prima por depreciación implícita en las transacciones de bonos del Ministerio de Hacienda (principal emisor público en República Dominicana) a través de la estimación inter-temporal de la curva de rendimiento de los mismos. Se encuentra que en el periodo analizado (septiembre 2012-diciembre 2016) la depreciación implícita en el mercado de bonos es mayor a la del mercado bancario, pero con menos volatilidad. Se verifica también, a través de la estimación de un modelo de vectores de corrección de errores, que existe una relación de largo plazo entre ambos indicadores de depreciación y que, a medida que ocurren desequilibrios entre ambos, es el mercado de bonos el que se ajusta, en un plazo de dos meses. Estos hallazgos indican que el mercado de bonos gubernamentales dominicanos tiene cierto nivel de eficiencia; sin embargo, aún existen oportunidades de arbitraje en el corto plazo.
\end{abstract}

Palabras clave: tipo de cambio; tasa de interés; mercado de capitales; bonos gubernamentales.

Códigos del JEL: E43, E44, F31, F41

* Analytica. Dirección: Calle Boy Scouts No. 16, Santo Domingo, República Dominicana. Teléfono: 1-809-885-7775. Agradecimientos: Agradezco a Jacqueline Mora, cuyo apoyo durante mi proceso de formación académica y profesional no puede ser resaltado lo suficiente, y a Joelle García por sus comentarios sobre el documento durante el proceso de redacción. 


\title{
The relationship between the implicit depreciation in the Government bond market and the banking bond market in the Dominican Republic
}

\author{
By EnRiQue Penson*
}

Submitted: 1/29/2017 - Accepted: 8/1/2017

\begin{abstract}
In this article, I estimate the causal relationship between the depreciation of the Dominican Peso vs. the US Dollar implicit in the transactions of government bonds in the bond market and the effective depreciation of the exchange rate reflected in commercial banking operations. To do this one extracted the bonus for depreciation implicit in transactions of bonds issued by Ministerio de Hacienda (biggest government debt issuer in the Dominican Republic) by estimating their yield curve intertemporally. One finds that, in the period analyzed (September 2012 to December 2016), the implicit depreciation in the bond market is both higher and less volatile than the one reflected in commercial banking operations. Also, it's verified, via the estimation of a vector error correction model, that a long-run relationship between both exists, and that when there is a disequilibrium between the two, it is the bond market that corrects itself, with a lag of two months. These findings indicate that the Dominican capital market has a certain level of efficiency; however, opportunities for arbitrage still exist in the short-run.
\end{abstract}

Keywords: exchange rate; interest rate; capital market; government bonds.

JEL Codes: E43, E44, F31, F41

* Analytica. Address: Calle Boy Scouts No. 16, Santo Domingo, República Dominicana. Telephone: 1-809-885-7775. Acknowledgements: I am thankful to Jacqueline Mora, whose support during my professional and academic preparation cannot by highlighted enough, and to Joelle García for her comments during the process of elaboration of this document. 


\section{Introducción}

El propósito de este trabajo es profundizar la investigación sobre la depreciación del peso dominicano frente al dólar estadounidense y su relación con el mercado de capitales de la República Dominicana. Este objetivo es particularmente importante por dos razones: primero, debido a la estrecha relación comercial que existe entre República Dominicana y Estados Unidos; esto agregado a la utilización de la moneda estadounidense como medio de cambio y depósito de valor en la economía dominicana hace que la formulación de perspectivas sobre la trayectoria de esta variable sea esencial para la toma de decisiones informadas por parte de los agentes económicos. Segundo, la activación del mercado de capitales dominicano durante los últimos años abre una nueva rama de investigaciones económicas que permite empezar a estudiar, entre otros temas, el nivel de eficiencia del mercado y la posible existencia de oportunidades de arbitraje.

Posteriormente, se probará que existe una relación de largo plazo entre la depreciación del tipo de cambio implícita en las transacciones del mercado de bonos emitidos por el Ministerio de Hacienda y la depreciación del tipo de cambio en el mercado bancario. Esto, inicialmente, es un indicio de cierta eficiencia en el mercado; sin embargo, también se verificará que en el corto plazo existen oportunidades de arbitraje en la medida que los desequilibrios entre ambas medidas de depreciación no se corrigen de inmediato.

El estudio actual contrasta con investigaciones anteriores en el hecho de que no busca identificar la totalidad de los factores explicativos de la variación del tipo de cambio o establecer su relación de equilibrio con los fundamentos económicos, lo cual es normalmente el objetivo principal de investigaciones sobre esta variable en el país. En su lugar, el presente artículo intenta lograr dos objetivos: primero, iniciar en el país el trabajo de modelación efectiva de los factores subyacentes que determinan las curvas de rendimiento en el mercado de capitales dominicano; Segundo, estimar la posibilidad y, por consiguiente, 
probar la existencia de oportunidades de arbitraje en el mercado de bonos dominicano a través de desequilibrios entre el mercado de capitales y el mercado bancario de divisas y la identificación de la velocidad en que los mismos se corrigen.

El resto de este documento se ordena de la siguiente forma: la próxima sección describe la literatura internacional y nacional relevante al tema en cuestión. La siguiente discute la data y la metodología de trabajo utilizada en el estudio. Posteriormente, se presentan las pruebas y los resultados econométricos. Finalmente, se cierra con una conclusión que resume los hallazgos y contempla la posibilidad de futuras investigaciones que tomen este documento como punto de partida.

\section{Revisión de la literatura}

La literatura relevante para esta investigación se compone de dos bloques notables. El primero de ellos comprende las publicaciones que indagan sobre la identificación, modelación teórica, estimación econométrica y predicción de la estructura de las tasas de interés y/o curvas de rendimiento. El segundo bloque se compone por la literatura que abarca el estudio del comportamiento del tipo de cambio en República Dominicana y algunas experiencias internacionales. Ambos son necesarios en la medida que este trabajo pretende determinar nuevos factores dinámicos que explican el comportamiento del tipo de cambio en el mercado de capitales y el mercado bancario dominicano, utilizando metodologías desarrolladas para la modelación de estructuras de tasas de interés y/o curvas de rendimiento.

El estudio práctico sobre la estimación de la estructura de rendimientos en un mercado de capitales se remonta al trabajo seminal de Nelson \& Siegel (1987), quienes concibieron por primera vez una forma funcional, a la vez teóricamente consistente y prácticamente estimable, de las curvas de rendimiento. Estos autores encuentran una serie de estudios anteriores que hacen esfuerzos para modelar curvas 
de rendimientos, pero con poco éxito; las estimaciones utilizaban formas funcionales que eran computacionalmente difíciles de estimar o, en su defecto, no tenían la complejidad suficiente para explicar los diferentes fenómenos de los rendimientos a través del tiempo y a veces eran inconsistentes en valores extremos. Uno de sus aportes principales radica en que su estimación logra replicar las estructuras históricas de las curvas de rendimientos de los bonos gubernamentales estadounidenses, manteniendo así consistencia con la teoría del comportamiento de los rendimientos.

Posteriormente al trabajo de Nelson \& Siegel, Diebold \& Li (2006) adaptan su metodología, aportando una interpretación de los factores estimados como un vector de tres parámetros que varía inter-temporalmente, explicando conjuntamente la curva de rendimiento en todos sus plazos. También, comprueban que la predicción de estos tres factores con estructuras relativamente simples (modelos univariados y autoregresivos) produce resultados consistentemente buenos en comparación con el resto de las metodologías generalmente aceptadas hasta la fecha (2006).

El aporte relevante de Diebold \& $\mathrm{Li}$ a la literatura radica en que se determinó que no solamente la curva de rendimiento puede ser modelada de forma parsimoniosa con una cantidad reducida de parámetros, sino que también estos pueden estimarse para varios plazos e interpretarse como series de tiempo dinámicas. Aquí radica una de las dos principales fuentes de inspiración para el documento presente: es posible extraer un factor más (la depreciación implícita del tipo de cambio) de la estructura de curvas de rendimiento si se conoce suficiente cantidad de transacciones en el mercado de capitales a diversos plazos y, a la vez, en la moneda local y la moneda extranjera.

La modelación econométrica del tipo de cambio en República Dominicana se ha concentrado principalmente en su impacto en otras variables macroeconómicas. Uno de los primeros trabajos de la historia moderna (post-2000) sobre el tema fue realizado por Sánchez (2006), que estimó el efecto del tipo de cambio sobre el ingreso y los 
precios. En la historia reciente, en algunos trabajos como aquel hecho por Michel (2015) se estimó el efecto del tipo de cambio sobre la inflación general de precios utilizando mejores fuentes de datos (mayor extensión de tiempo en las mismas). En otras investigaciones como la de Cruz-Rodríguez (2015) se ha encontrado que la depreciación del tipo de cambio real afecta negativamente al crecimiento económico, aun considerando múltiples medidas de desalineación real de la depreciación del peso dominicano frente al dólar estadounidense.

Sobre los determinantes del tipo de cambio propiamente dicho se encuentra trabajos como los de la autoría de Andújar \& Medina (2008), que proponen un modelo de proyección del tipo de cambio que a largo plazo depende del diferencial de tasas de interés e inflaciones, y una medida de riesgo cambiario. Notan, sin embargo, que la estimación arroja un coeficiente al diferencial de tasas de interés (variable más relevante en este documento) que implicaría que en la medida que las tasas locales aumentan por encima de la referencia internacional (EE.UU.), el tipo de cambio se depreciaría, lo que contrasta con la teoría económica. En el mismo documento señalan que este "rompecabezas de la relación tasa de interés-tipo de cambio en RD ha sido notado por otros autores..." (p. 196).

En contraste con la literatura dominicana (donde existía hasta hace poco una importante ausencia de fuentes de datos extensas sobre las transacciones del mercado de valores), en el ámbito internacional sí se ha estudiado de manera desarrollada la relación entre el tipo de cambio y los rendimientos de los bonos. Un ejemplo de esto es el trabajo de Gadanecz, Miyama \& Shu (2014), que prueba que existe una relación de causalidad desde las variaciones en el tipo de cambio hacia el rendimiento de los bonos en moneda local de veinte países ${ }^{1}$ considerados mercados emergentes; esto implícitamente indicaría que dentro de los bonos locales de cada uno de estos países existe un componente atado

\footnotetext{
${ }^{1}$ Brasil, Chile, China, Colombia, República Checa, Hong Kong SAR, Hungría, India, Indonesia, Israel, Corea, Malaysia, México, Filipinas, Polonia, Rusia, Singapur, Sudáfrica, Tailandia, Turquía.
} 
a la depreciación implícita del tipo de cambio, aumentando así el rendimiento de los bonos cuando se espera una mayor depreciación.

La combinación de la literatura internacional sobre los determinantes de las curvas de rendimiento, que permite concebir la extracción de factores dentro de las mismas y estimar las relaciones causales desde el tipo de cambio hacia el rendimiento expresado en estas curvas, con las nuevas fuentes de datos dominicanas que facilitan las transacciones de bonos gubernamentales, favorece la propuesta de poder estimar una posible causalidad (quizás en ambas direcciones) entre el rendimiento de estos bonos y el tipo de cambio.

\section{Datos y metodología}

La fuente de datos utilizada se obtuvo a través de la Dirección General de Crédito Público de la República Dominicana, que ofrece al público en general, a través de su página web, todas las transacciones registradas de los bonos emitidos por el Ministerio de Hacienda. Los datos transaccionales existen desde el año 2009, pero solo a partir del año 2012 el volumen de transacciones incrementa hasta niveles que, posteriormente, se verificará permiten inferencia estadística.

En la figura 1 se observa el volumen transado de los bonos del Ministerio de Hacienda en el mercado de bonos dominicano. Se percibe que ocurren dos períodos de alta aceleración del valor transado: 2012-2013 y 2014-2015.

La estrategia de identificación consiste primero en determinar la depreciación esperada implícita en las transacciones de los bonos del Ministerio de Hacienda. Para hacer esto se asumen cuatro hechos estilizados sobre la forma en que se formula la estructura de rendimientos en el mercado de bonos dominicano:

1. Existe un nivel de rendimiento mínimo demandado por los inversionistas, aun para instrumentos con madurez cercana al vencimiento o en la fecha de vencimiento. 
Figura 1. Valor Transado de los bonos del Ministerio de Hacienda

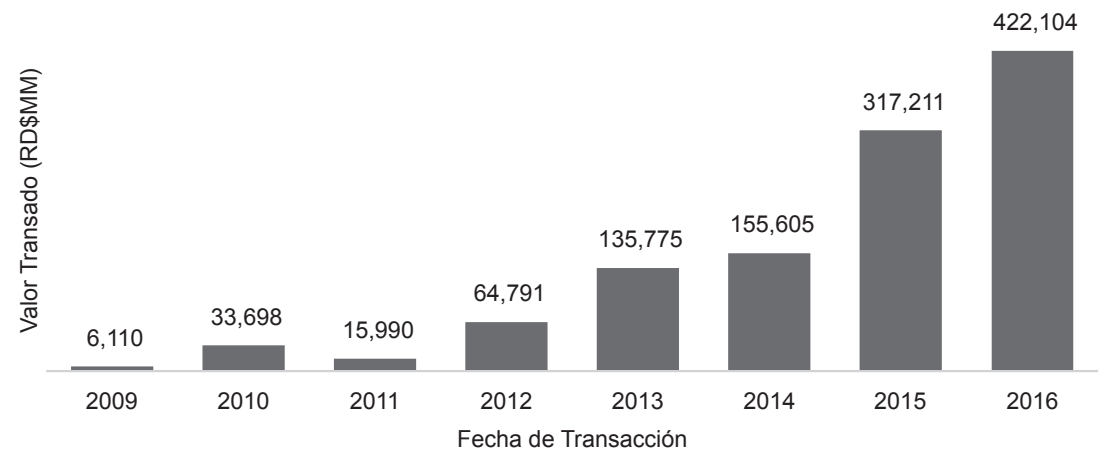

Notas. Esta figura muestra la evolución del valor transado (en millones de pesos dominicanos) en bonos del Ministerio de Hacienda en el período comprendido entre los años 2009-2016, construido con datos obtenidos a partir de la Dirección General de Crédito Público de la República Dominicana.

2. Existe una relación significativa y usualmente positiva entre el rendimiento de un bono y su madurez (diferencia entre la fecha de vencimiento y la fecha de la transacción que se observa).

3. La forma de la relación entre el rendimiento y la madurez de un bono presenta una convexidad; en la medida que la madurez de un bono hipotético aumenta, su rendimiento cada vez incrementa en menor magnitud.

4. Existe una diferencia significativa entre el rendimiento de los bonos transados en pesos y los transados en dólares, aun luego de controlar por la madurez de los mismos; esta diferencia se puede interpretar como una depreciación implícita del peso o una expectativa de depreciación. 
Figura 2. Rendimientos por madurez en las transacciones de bonos de Hacienda.

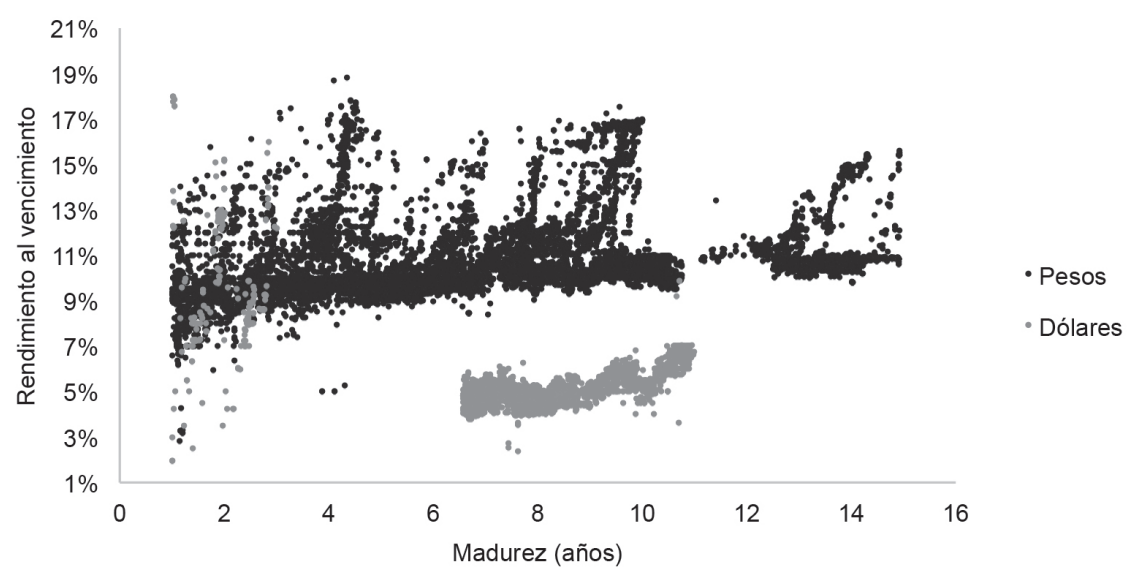

Notas. Esta figura muestra como un punto cada transacción de bonos del Ministerio de Hacienda en el año 2016, ubicándola según su madurez y rendimiento al vencimiento. La moneda en la que el bono está denominado se encuentra en la leyenda. Se filtraron las transacciones de bonos con madurez menor a un año debido a que ciertos tipos de operaciones de corto plazo en el mercado ocurren a precios (y por tanto rendimientos) que, primero, no son representativos del mercado, y segundo, se encuentran por fuera del comportamiento normal del mismo.

Utilizando la base de datos transaccional mencionada anteriormente, se estimó un modelo simple de la curva de rendimientos con la siguiente estructura:

$$
r=a_{0}+a_{1} * \text { pesos }+a_{2} * \log (\text { madurez })
$$

Donde:

$r=$ Rendimiento al vencimiento del bono.

madurez $=$ Diferencia en años entra la fecha de operación de la transacción y el vencimiento del bono.

peso = Variable dicotómica que toma valor " 1 " cuando el bono está denominado en pesos y " 0 ” cuando lo está en dólares. 
La especificación de la ecuación 1 propone que el rendimiento al vencimiento de un bono depende de tres factores. Primero, existe un nivel mínimo de rendimiento $\left(\mathrm{a}_{0}\right)$, el cual es equivalente al rendimiento al vencimiento de un bono de Hacienda en dólares a punto de vencer (con madurez cero o cercana a cero); un aumento de este nivel mueve la curva de rendimiento completa de forma paralela hacia arriba. El segundo coeficiente $\left(a_{1}\right)$ indica la prima que demandaría un inversionista en el mercado de bonos para comprar un bono de Hacienda denominado en pesos, en contraste con uno en dólares; el valor estimado de este coeficiente revela la depreciación implícita, o esperada, en el mercado de bonos. El tercer coeficiente denota el aumento en el rendimiento de un bono del Ministerio de Hacienda demandado en el mercado por cada $1 \%$ que aumente la madurez (expresada en años) del mismo.

La estrategia de identificación de esta curva de rendimiento, aunque simple, apunta a lograr un primer acercamiento al problema de estimación. La escasez de volumen de transacciones en el mercado no permite estimar de forma eficaz formas funcionales más flexibles de la estructura de las tasas de interés, como las de la metodología de Nelson \& Siegel (1987), que son las más comúnmente utilizadas en estudios que descomponen los factores determinantes en la estructura de una curva de rendimientos.

Con este modelo se estimó de forma individual la estructura de la curva de rendimiento del mercado de bonos del Ministerio de Hacienda para todos los meses (observaciones) en la muestra (94), de los cuales en 52 casos consecutivos (septiembre 2012 en adelante) fue posible identificar exitosamente los parámetros de la ecuación descrita con un coeficiente de poder explicativo (R2 ajustado) suficientemente alto (mayor a 80\%). Esto se confirma en la figura debajo, que muestra el resultado de este coeficiente para todas las fechas de estimación. 
Figura 3. Poder explicativo de las curvas de Rendimiento estimadas

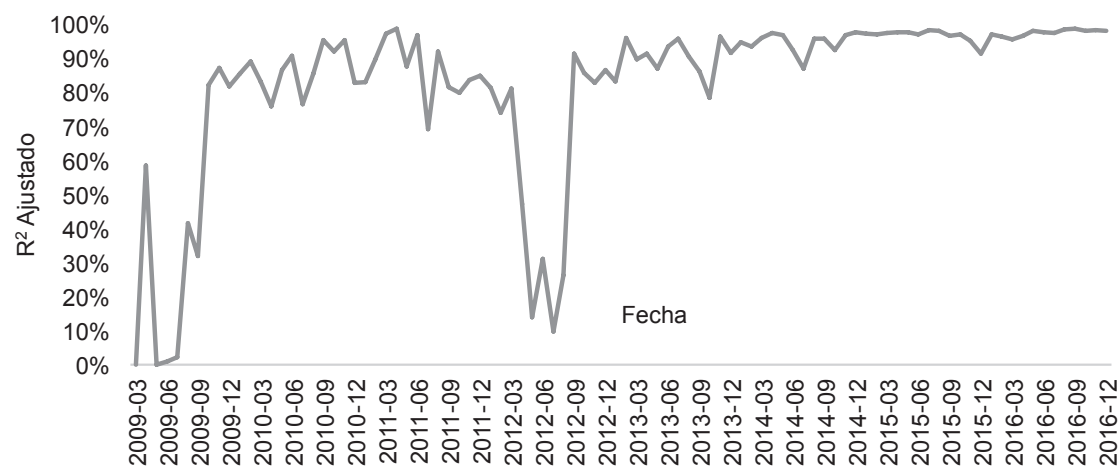

Notas. Esta figura muestra la evolución del R2 ajustado (poder explicativo) de la ecuación 1, estimada para todos los meses entre los años 2009 y 2016.

Debajo se visualizan los tres parámetros estimados, notándose que desde el año 2012 el estimador asociado a la denominación en pesos se ha mantenido estable con cierta disminución, al igual que la prima por madurez. En contraste, el valor del intercepto ha incrementado hasta estabilizarse en 2016. La interacción de un intercepto aumentando y una prima por madurez disminuyendo implica un aplanamiento de la curva de rendimientos de los bonos gubernamentales, permitiendo que el Estado se financie a mayores plazos sin incurrir un significativo aumento en su costo de capital; análogamente, esto revela que en el período analizado los inversionistas en el mercado de capitales dominicano cada vez demandan una menor prima por el riesgo asociado a la madurez, indicando quizás cierto nivel de confianza en la capacidad de pago del Estado dominicano en el mediano y largo plazo.

Luego, se construyeron dos series de tiempo mensuales desde septiembre de 2012. La primera es igual al parámetro estimado de la variable dicotómica "pesos" $\left(\mathrm{a}_{1}\right)$ que, efectivamente, muestra la depreciación implícita (o esperada) en el mercado de bonos de 
Hacienda; la segunda es igual a la depreciación interanual del tipo de cambio venta promedio de entidades financieras reportado por el Banco Central de la República Dominicana.

Figura 4. Parámetros estimados para la eCuación 1 por fecha

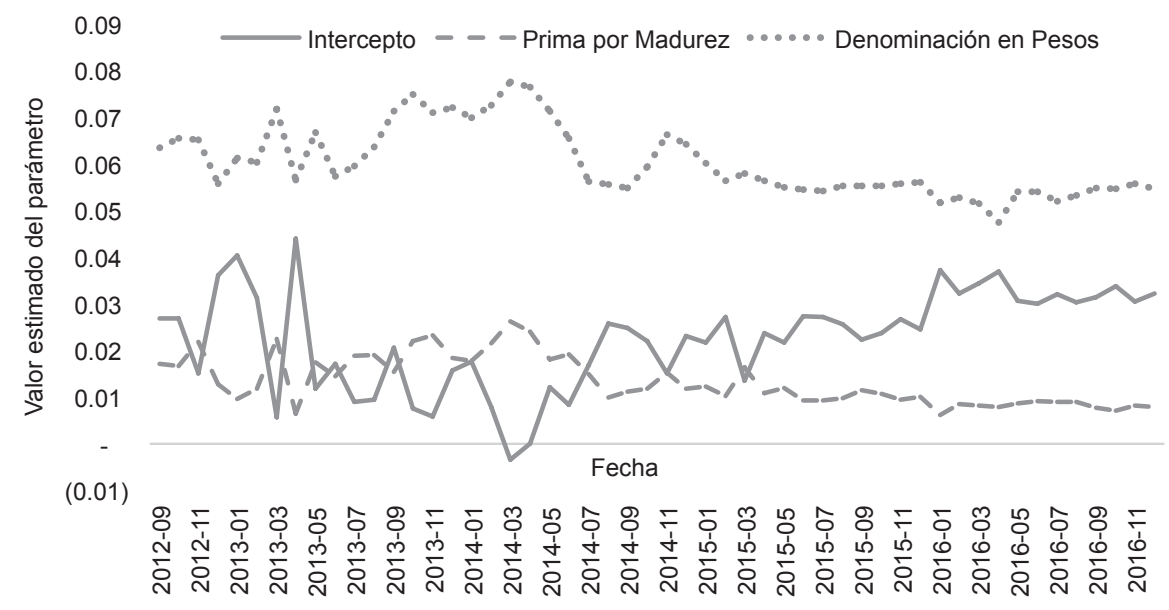

Notas. Esta figura muestra la evolución del valor estimado para los parámetros de la ecuación 1 sobre cada mes entre septiembre 2012 y diciembre 2016. El intercepto corresponde al parámetro, la denominación en pesos al parámetro, y la prima por madurez al parámetro.

En la figura 4, se nota debajo que la depreciación implícita en el mercado de bonos se ha mantenido por encima y con mayor estabilidad que la depreciación del tipo de cambio peso/dólar en el mercado bancario (excluyendo el período junio-octubre de 2013). Esto sugiere que, a pesar del movimiento efectivo del tipo de cambio reflejado en las transacciones del mercado bancario, los inversionistas en el mercado de capitales mantienen expectativas de depreciación relativamente ancladas.

En la tabla 1 (debajo) se verifica que la depreciación implícita en el mercado de bonos mantiene a la vez una media por encima de la del mercado bancario, pero una volatilidad significativamente menor, lo que también se muestra a través del hecho de que los valores mínimos 
y máximos de la asociada al mercado bancario son más extremos que los asociados al mercado de bonos.

Figura 5. Depreciación interanual del peso dominicano frente al dólar ESTADOUNIDENSE

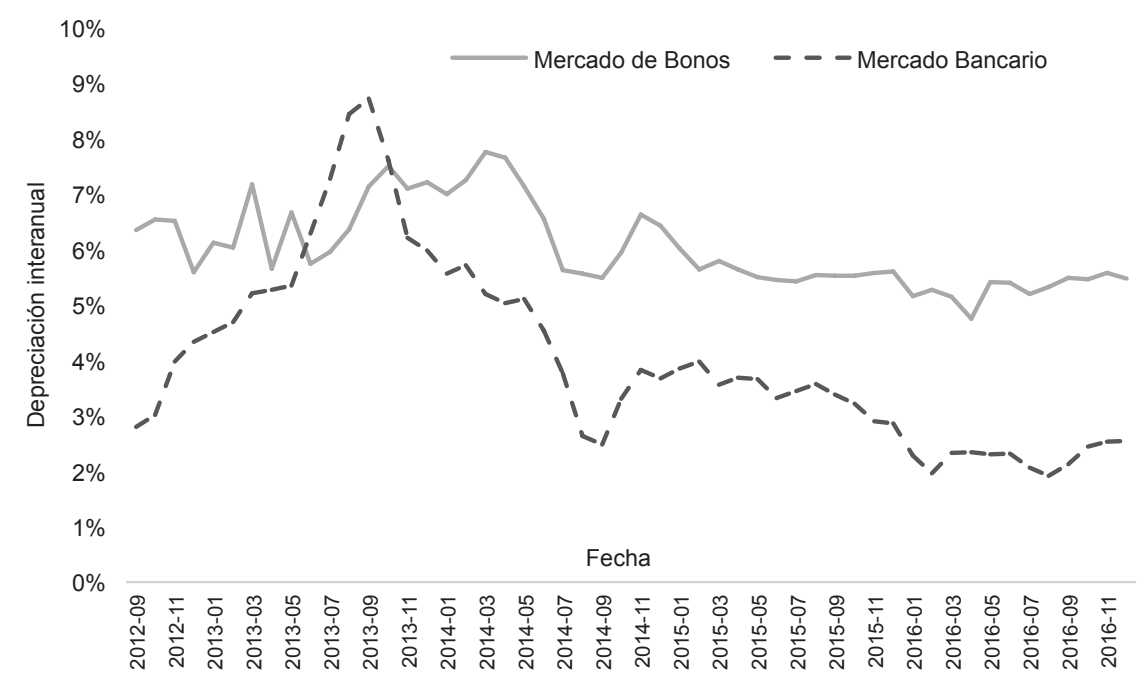

Notas. Esta figura muestra la evolución de las dos medidas de la depreciación interanual del tipo de cambio peso/dólar en el período septiembre 2012 - diciembre 2016.

Tabla 1. Resumen estadístico de ambas Series de Depreciación Utilizadas

\begin{tabular}{cccccc}
\hline Variable & Observaciones & Media & Desviación Estándar & Mínimo & Máximo \\
\hline Mercado de bonos & 52 & $6.03 \%$ & $0.75 \%$ & $4.74 \%$ & $7.76 \%$ \\
Mercado bancario & 52 & $4.02 \%$ & $1.68 \%$ & $1.92 \%$ & $8.72 \%$ \\
\hline
\end{tabular}

Notas. Esta tabla muestra estadísticas descriptivas de las dos series indicativas de la depreciación del tipo de cambio; la primera es igual a la estimación del parámetro correspondiente al peso en la ecuación 1 (a1), que indica la depreciación implícita en el mercado de bonos, y la segunda es igual a la depreciación interanual del tipo de cambio de venta de las entidades financieras promedio mensual. El período de cálculo comprende desde septiembre de 2012 hasta diciembre de 2016.

La estrategia de estimación implica la formulación simultánea de un modelo de largo plazo y uno de corto plazo, lo que se conoce en la literatura como un modelo de vectores de corrección del error 
(VECM, por sus siglas en inglés). El mismo propone que existe una relación de largo plazo que determina el comportamiento de algunas variables, pero que en el corto plazo pueden desviarse de este nivel de equilibrio y tienden hacia el equilibrio de nuevo en cierto período de tiempo.

Con las dos series de tiempo (depreciación implícita en el mercado de bonos y explícita en el mercado bancario) se estima una ecuación de largo plazo con la siguiente estructura:

$$
t_{\text {bonos }_{t}}=b_{0}+b_{1} * \text { tc }_{\text {bancario }}+e_{t}
$$

Donde:

$t c_{\text {bonos }}=$ Depreciación implícita en el mercado de bonos en el período t.

$t c_{\text {bancario }_{t}}=$ Depreciación explícita en el mercado bancario en el período t.

La ecuación 2 sugiere una relación de largo plazo entre la depreciación implícita en el mercado de bonos y la depreciación reflejada en el mercado bancario; en la medida que ambos mercados sean eficientes esta relación debe ser significativa. El parámetro asociado a la constante en el modelo $\left(\mathrm{b}_{0}\right)$ explica cuánto por encima (o debajo) de la depreciación en el mercado bancario se ubica, en promedio, la depreciación explícita en el mercado de bonos; debido a los resultados de la tabla 1, se espera que el signo de este parámetro sea positivo. El segundo estimador $\left(b_{1}\right)$ explica cuánto aumenta la depreciación implícita en el mercado de bonos por cada punto porcentual que aumente la del mercado bancario, en el largo plazo.

Luego, se extrajo el residuo (error de estimación $=e_{t}$ ) de la ecuación anterior, que se consideraría el desequilibrio de largo plazo, y 
se introdujo en dos ecuaciones en diferencias de corto plazo con la siguiente forma funcional:

$$
\begin{aligned}
& \Delta\left(\text { tc }_{\text {bonos }_{t}}\right)=c_{10}+c_{11} * e_{t-1}+c_{12} * \Delta\left(\text { tc }_{\text {bonos }_{t-1}}\right)+c_{13} * \Delta\left(\text { tc }_{\text {bonos }_{t-2}}\right)+c_{14} \\
& * \Delta\left(\text { tc }_{\text {bancario }_{t-1}}\right)+c_{15} * \Delta\left(\text { tc }_{\text {bancario }_{t-2}}\right)+v_{t} \\
& \Delta\left(\text { tc }_{\text {banario }_{t}}\right)=c_{20}+c_{21} * e_{t-1}+c_{22} * \Delta\left(\text { tc }_{\text {bancario }-1}\right)+c_{23} \\
& * \Delta\left(\text { tc }_{\text {bancario }_{t-2}}\right)+c_{24} * \Delta\left(\text { tc }_{\text {bonos }_{t-1}}\right)+c_{25} * \Delta\left(\text { tc }_{\text {bonos }_{t-2}}\right) \\
& +\gamma_{t}
\end{aligned}
$$

\section{Pruebas y resultados econométricos}

La estimación del modelo de vectores de corrección de errores (VECM) requiere, inicialmente, una confirmación de que la relación de largo plazo no es debido, únicamente, a la consistencia estadística. Para esto se realiza una prueba de cointegración de Johansen, mostrada debajo, que identifica si efectivamente existe una relación de largo plazo entre ambas variables, determinándose que se mueven conjuntamente en largos períodos de tiempo. La tabla 2 muestra los resultados de esta prueba, que indican que existe por lo menos una ecuación de largo plazo estimable entre ambas variables.

Luego, es necesario encontrar la cantidad de rezagos de las variables que es propicio incluir en el modelo estimado. A través de diferentes criterios de información se identifica que el VECM debe ser estimado con dos o tres rezagos de las variables explicativas. Se eligió una estimación con tres rezagos debido a que resultados de investigaciones econométricas como la realizada por Gonzalo (1994) indican que existe un riesgo significativo de sub-especificar el número de residuos en un VECM, lo que conduce a la presencia de correlación serial. 
Tabla 2. Prueba de cointegración de Johansen sobre ambas Series indicativas de DEPRECIACIÓN

\begin{tabular}{cccccc}
\hline Orden máximo & Parámetros & LL & Eigenvalue & Estadístico Traza & Valor crítico (5\%) \\
\hline 0 & 10 & 397.69 &. & 15.8574 & 15.41 \\
1 & 13 & 404.90 & 0.25499 & $1.4341^{*}$ & 3.76 \\
2 & 14 & 405.62 & 0.02884 & & \\
\hline
\end{tabular}

Notas. Esta tabla muestra los resultados de la prueba de cointegración de Johansen entre las dos medidas de la depreciación del peso contempladas. Indica, con un asterisco “*”, que existe por lo menos una ecuación de cointegración significativa (con un $95 \%$ de confianza) entre ambas variables.

TABLA 3: CRITERIOS DE INFORMACIÓN DEL VECM PROPUESTO CON DIFERENTES REZAGOS

\begin{tabular}{ccccccccc}
\hline Rezagos & LL & LR & df & $p$ & FPE & AIC & HQIC & SBIC \\
\hline 0 & 311.463 & & & & $8.60 \mathrm{E}-09$ & -12.8943 & -12.8648 & -12.8163 \\
1 & 385.612 & 148.3 & 4 & 0.000 & $4.60 \mathrm{E}-10$ & -15.8172 & -15.7288 & -15.5833 \\
2 & 393.766 & $16.307^{*}$ & 4 & 0.003 & $3.90 \mathrm{E}-10$ & -15.9902 & $-15.8429 *$ & $-15.6004^{*}$ \\
3 & 398.019 & 8.5067 & 4 & 0.075 & $3.9 \mathrm{E}-10^{*}$ & $-16.0008^{*}$ & -15.7946 & -15.455 \\
4 & 401.288 & 6.5367 & 4 & 0.162 & $4.00 \mathrm{E}-10$ & -15.9703 & -15.7051 & -15.2686 \\
\hline
\end{tabular}

Notas. La tabla muestra el valor de diferentes criterios de información para la estimación del VECM con diferentes rezagos. El asterisco (*) denota el valor más significativo para cada criterio de información, lo que es un indicador del número de rezagos que debe utilizarse en el modelo final.

Al estimar el VECM, los resultados de la tabla 4 (debajo) señalan que es la depreciación implícita en el mercado de bonos que se corrige a la del mercado bancario; esto se verifica en el coeficiente atado a la variable "Error de Largo Plazo (t-1)", que es el residuo de la ecuación de largo plazo rezagado un período, y la magnitud del mismo explica que los desequilibrios de largo plazo se corrigen aproximadamente en dos meses $(1 / 0.53=1.9)$. Esta corrección es significativa con un $99 \%$ de confianza, rechazando la hipótesis nula de que el parámetro sea estadísticamente igual a cero. En el caso del mercado bancario, se nota que el coeficiente de corrección de error no es estadísticamente diferente de cero, indicando que es el mercado de bonos que se corrige a los movimientos del tipo de cambio en el mercado bancario. La velo- 
cidad de corrección, alrededor de dos meses, abre espacio para oportunidades de arbitraje por parte de los agentes económicos; en la medida que esta brecha sea aprovechada, se espera que la velocidad aumente (duración disminuya) hasta un nivel operacionalmente mínimo.

TABla 4: Resultados de la estimación del VECM

\begin{tabular}{crr}
\hline & $\Delta$ (Dep. Bonos) & $\Delta($ Dep. Bancos $)$ \\
\hline Error de Largo Plazo (t-1) & $(0.53)$ & $(0.10)$ \\
$\Delta$ (Dep. Bonos) (t-1) & - & 0.50 \\
& $0.04)$ & $(0.01)$ \\
$\Delta($ Dep. Bonos) $(\mathrm{t}-2)$ & 0.78 & 0.93 \\
& 0.07 & $(0.14)$ \\
$\Delta$ (Dep. Bancos) $(\mathrm{t}-1)$ & $(0.04)$ & 0.30 \\
& 0.80 & 0.61 \\
$\Delta($ Dep. Bancos) $(\mathrm{t}-2)$ & $(0.07)$ & - \\
& 0.64 & $(0.27)$ \\
Constante & 0.00 & 0.07 \\
& 0.94 & $(0.00)$ \\
$\mathrm{N}$ & 49 & 0.70 \\
$\mathrm{R}^{2}$ & 0.38 & 49 \\
\hline
\end{tabular}

Notas. Esta tabla muestra los resultados de la estimación del VECM. Se muestran los parámetros estimados, con el nivel de significancia asociada al parámetro estimado debajo (en itálicos) para cada variable. La hipótesis nula para cada estimador es que sea igual a cero. Debajo se nota el número de observaciones en la regresión $(\mathrm{N})$ y el indicador de ajuste estadístico (R2).

Como forma de verificar la validez de los resultados, se obtuvieron los valores propios asociados a la estabilidad del VECM (tabla 5), que se mantuvieron dentro de los límites de lo necesario para que el modelo tenga estabilidad dinámica. También, se realizó una prueba del multiplicador de Lagrange (tabla 6), a través de la cual no fue posible rechazar la hipótesis nula de no correlación serial de los residuos. Finalmente, se comprobó que no se rechaza la hipótesis nula de que los residuos tienen una distribución normal a través de pruebas de Jarque-Bera, asimetría y kurtoris (tablas 7-9). 
Tabla 5. Modulus del VECM

\begin{tabular}{c}
\hline Modulus estimado \\
1.00 \\
0.70 \\
0.70 \\
0.65 \\
0.49 \\
0.49 \\
\hline
\end{tabular}

Notas. Modulus estimados del VECM, que confirman la condición de estabilidad de los valores propios del VECM. Esta condición se cumple en la medida que los valores en la tabla no superen la unidad.

Tabla 6. Prueba del multiplicador de Lagrange sobre los residuos del VeCM

\begin{tabular}{cccc}
\hline Rezago & Chi Cuadrado & df & Prob $>$ Chi Cuadrado \\
1 & 2.6838 & 4 & 0.61206 \\
2 & 0.7326 & 4 & 0.94725 \\
\hline
\end{tabular}

Notas. Esta tabla muestra los resultados de la prueba del multiplicador de Lagrage sobre los residuos del VECM estimado, notándose que no se rechaza la hipótesis nula de no correlación serial en los residuos.

Tabla 7. Prueba de Jarque-Bera sobre los residuos del VECM

\begin{tabular}{cccc}
\hline Ecuación & Chi Cuadrado & df & Prob $>$ Chi Cuadrado \\
$\Delta$ (Dep. Bonos) & 0.947 & 2 & 0.62295 \\
$\Delta$ (Dep. Bancos) & 1.286 & 2 & 0.5258 \\
Ambas & 2.232 & 4 & 0.69313 \\
\hline
\end{tabular}

Notas. Esta tabla muestra los resultados de la prueba de Jarque-Bera sobre los residuos del VECM estimado, notándose que no se rechaza la hipótesis nula de normalidad en los residuos. 
Tabla 8. Prueba de asimetría sobre los residuos del VeCM

\begin{tabular}{ccccc}
\hline Ecuación & Asimetría & Chi Cuadrado & df & Prob $>$ Chi Cuadrado \\
$\Delta$ (Dep. Bonos) & 0.16698 & 0.228 & 1 & 0.63323 \\
$\Delta$ (Dep. Bancos) & -0.3967 & 1.285 & 1 & 0.25693 \\
Ambas & & 1.513 & 2 & 0.46933 \\
\hline
\end{tabular}

Notas. Esta tabla muestra los resultados de la prueba de asimetría sobre los residuos del VECM estimado, notándose que no se rechaza la hipótesis nula de no asimetría en los residuos.

Tabla 9. Prueba de Kurtosis sobre los Residuos del VECM

\begin{tabular}{ccccc}
\hline Ecuación & Kurtosis & Chi Cuadrado & df & Prob $>$ Chi Cuadrado \\
$\Delta$ (Dep. Bonos) & 3.5934 & 0.719 & 1 & 0.39651 \\
$\Delta$ (Dep. Bancos) & 2.9852 & 0 & 1 & 0.98317 \\
Ambas & & 0.719 & 2 & 0.69791 \\
\hline
\end{tabular}

Notas. Esta tabla muestra los resultados de la prueba de kurtosis sobre los residuos del VECM estimado, notándose que no se rechaza la hipótesis nula de no kurtosis en los residuos.

\section{Conclusión}

En esta investigación se comprobaron cuatro características importantes de la depreciación del tipo de cambio en República Dominicana. Primero, los agentes económicos que transan en el mercado de capitales asignan una prima a los bonos en pesos que supera la depreciación efectiva en el mercado bancario de divisas y esta "expectativa de depreciación" presenta signos de estar anclada, con la mitad de la volatilidad del mercado bancario. Segundo, existe una relación de largo plazo entre la depreciación implícita en el mercado de bonos y la efectiva en el mercado bancario, lo que refleja cierto nivel de eficiencia en el mercado dominicano, indicando que los agentes económicos que transan en el mercado de bonos incorporan los cambios en el tipo de cambio al discriminar entre los bonos del Ministerio de Hacienda denominados en dólares y los denominados en pesos. Tercero, existen desequilibrios en esta relación de corto plazo que permiten oportuni- 
dades de arbitraje por parte de los agentes económicos. Cuarto, y último, los desequilibrios en el mercado se corrigen a través del mercado de bonos con relativa rapidez (casi dos meses) y se espera que, en la medida que el mercado de capitales se profundice, esta corrección ocurra en un menor plazo.

Posteriores investigaciones deben expandir, entre diversos aspectos, sobre dos principales: primero, en la medida que el volumen de transacciones del mercado incremente, debe ser posible estimar formas funcionales más flexibles de las curvas de rendimiento del mercado. Segundo, es necesario incorporar la dinámica entre el mercado bancario y el mercado de capitales en los modelos económicos de proyección, que apuntan a formar perspectivas sobre el desempeño futuro de las variables económicas, con el propósito de afinar las mismas y facilitar la toma de decisiones de negocios e inversión a la vez oportunas y fundamentadas en mejores predicciones económicas.

\section{Referencias}

Akaike, H. (1976). Canonical Correlation Analysis of Time Series and The Use of An Information Criterion. Systems identification: Advances and Case Studies, New York: Academic Press, 27-96.

Andújar, J. \& Medina, A. (2008). Un modelo macroeconómico de pequeña escala para la República Dominicana. Modelos macroeconométricos de la banca central: Centroamérica y República Dominicana, México: Comisión Económica para América Latina y el Caribe, 179-219.

Breusch, T. y Pagan A. (1980). The Lagrange Multiplier Test and its Applications to Model Specification in Econometrics. Review of Economic Studies, 47(1): 239-253. 
Cruz-Rodríguez, A. (2015). Crecimiento económico y desalineación del tipo de cambio real en la República Dominicana: ¿hay alguna relación? Ciencia y Sociedad, 40(3): 571-605.

Dickey, D. y Fuller W. (1981). Likelihood ratio statistics for autoregressive time series with a unit root. Econometrica, 49(4): 1057-1077.

Diebold, F. \& Li, C. (2006). Forecasting the Term Structure of Government Bond Yields. Journal of Econometrics, 130: 337-364.

Engel, R. y Granger C. (1987). Cointegration and Error Correction: Representation, Estimation and Testing. Econometrica, 55(2): 251-276.

Gadanecz, B., Miyajima, K. \& Shu, C. (2014). Exchange rate risk and local currency sovereign bond yields in emerging markets. Bank for International Settlements, No. 474. Basilea: Bank for International Settlements.

Gonzalo, J. (1994). Five alternative methods of estimating long-run equilibrium relationships. Journal of Econometrics, 60(1-2): 203-233.

Granger, C. (2004). Time Series Analysis, Cointegration, and Applications. American Economic Review, 94(3): 421-425.

Hansen, H. y Johansen S. (1991). Some Tests for Parameter Constancy in Cointegrated VAR-models. The Econometrics Journal, 2(2): 306-333.

Jarque, C. y Bera A. (1987). A Test for Normality of Observations and Regression Residuals. International Statistical Review, 55(2): 163-172. 
Johansen, S. (1988). Statistical Analysis of Cointegrating Vectors. Journal of Economic Dynamics and Control. 12(2-3): 231-254.

Michel, J. (2015). Determinantes internos y externos de la inflación en una economía pequeña y abierta: el caso de la República Dominicana. Serie de Estudios Económicos, No. 11. Santo Domingo: Banco Central de la República Dominicana.

Nelson, C. y Siegel, A. (1987). Parsimonious Modeling of Yield Curves. The Journal of Business, 60(4): 473-489.

Phillips, P. y Perron, P. (1988). "Testing for unit roots in time series regression.” Biometrika, 75(2): 335-346.

Sánchez, J. (2006). Money, income, prices, and exchange rates in the Dominican Republic. Savings and Development, 30(1): 31-38.

Svensson, L. (1994). Estimating Forward Interest Rates with the Extended Nelson and Siegel Method. Sveriges Riksbank Quarterly Review, 1995(3): 13-26. 